\title{
NONLINEAR EVOLUTION OF BEAM-PLASMA INSTABILITY IN INHOMOGENEOUS MEDIUM
}

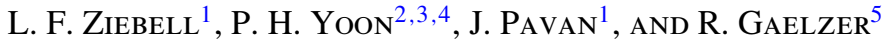 \\ ${ }^{1}$ Instituto de Física, UFRGS, Porto Alegre, RS, Brazil \\ 2 IPST, University of Maryland, College Park, USA \\ ${ }^{3}$ School of Space Research, Kyung Hee University, Yongin, Republic of Korea \\ ${ }^{4}$ MTL, Belmont, MA, USA \\ ${ }^{5}$ Instituto de Física e Matemática, UFPel, Pelotas, RS, Brazil \\ Received 2010 September 27; accepted 2010 November 9; published 2010 December 23
}

\begin{abstract}
The problem of electron-beam propagation in inhomogeneous solar wind is intimately related to the solar type II and/or type III radio bursts. Many scientists have addressed this issue in the past by means of quasi-linear theory, but in order to fully characterize the nonlinear dynamics, one must employ weak-turbulence theory. Available numerical solutions of the weak-turbulence theory either rely on only one nonlinear process (either decay or scattering), or when both nonlinear terms are included, the inhomogeneity effect is generally ignored. The present paper reports the full solution of weak-turbulence theory that includes both decay and scattering processes, and also incorporating the effects of density gradient. It is found that the quasi-linear effect sufficiently accounts for the primary Langmuir waves, but to properly characterize the back-scattered Langmuir wave, which is important for eventual radiation generation, it is found that both nonlinear decay and scattering processes make comparable contributions. Such a finding may be important in the quantitative analysis of the plasma emission process with application to solar type II and/or type III radio bursts.
\end{abstract}

Key words: instabilities - plasmas - solar wind - turbulence - waves

Online-only material: color figures

\section{INTRODUCTION}

The beam-plasma interaction is a fundamental problem in space and solar physics. It is well known that it is intimately related to the solar type II and type III radio bursts (Goldman 1983; Melrose 1985; Robinson \& Cairns 1998a, 1998b, 1998c). The radio emission mechanism involves the excitation of Langmuir $(L)$ waves by the beam-plasma instability and partial conversion of electrostatic wave energy associated with the Langmuir turbulence to electromagnetic wave energy of the radio bursts. The physics of the plasma emission is a complicated one, but it first involves nonlinear interactions between the electrons, Langmuir $(L)$ and ion-sound $(S)$ waves, as well as with the transverse electromagnetic waves.

The electron beam that excites Langmuir waves propagates in an inhomogeneous medium. In one of the earliest works, Vedenov et al. (1967) discussed the dynamical evolution of electron beams in spatially inhomogeneous plasmas. Classic papers by Takakura \& Shibahashi (1976) and by Magelssen \& Smith (1977) also discussed the problem of electron-beam propagation in inhomogeneous media. Later works by Grognard (1985) also addressed the same problem. More recent works by Kontar (2001), Li et al. (2002), and Khalilpour et al. (2009) solved similar problems. All the above-referenced discussions, however, included only quasi-linear physics.

Quasi-linear theory of beam-plasma instability in a uniform medium is a well-known problem. The numerical solutions of one-dimensional quasi-linear beam problem can be obtained routinely, and even higher-dimensional solutions are available in the literature (Appert et al. 1976; Ishihara \& Hirose 1981, 1983a, 1983b; Muschietti et al. 1997). The presence of density inhomogeneity makes the problem a bit more complicated. Nevertheless, the essential physics of the velocity space plateau formation and saturation of Langmuir waves are all wellunderstood features. In order to fully characterize the nonlinear dynamics of the beam-plasma interaction, one must go beyond quasi-linear theory and include other nonlinear processes.

One of the most useful theoretical tools that can be employed to investigate nonlinear evolution of the beam-plasma system is weak-turbulence theory. The essential theory was formulated by pioneers of modern plasma physics in the 1970s-for a recent reformulation of the problem, see Yoon (2006). Even though the basic theory was available in the literature, numerical solutions of the basic equations did not become available until quite recently. The first numerical solutions of the weakturbulence equation for beam-plasma instability was discussed by Muschietti \& Dum (1991). However, their discussion only included nonlinear scattering, but left out the decay term. The paper by Li et al. (2003), on the other hand, included only the decay process in their numerical solution but ignored the scattering process.

The numerical solution of the entire weak-turbulence equation including both decay and scattering processes was first obtained by Ziebell et al. (2001). Their one-dimensional numerical analysis was later extended to two dimensions in velocity space by Ziebell et al. (2008a, 2008b) and by Pavan et al. (2009). However, the numerical solutions obtained by these authors were applicable only to uniform plasmas. As noted, however, for solar and interplanetary applications, the spatial inhomogeneity cannot be ignored. In this regard, Kontar \& Pécseli (2002) presented a numerical analysis in one-dimensional velocity space, including a simplified treatment of a density inhomogeneity characterized by a scale length $L$, taking into account three-wave decay and scattering effects. Li et al. (2003, 2006a, 2006b) also provided solution to the weak-turbulence problem including spatial inhomogeneity, but they included only the decay term.

In the present paper, we study the time evolution of the beam-plasma instability in an inhomogeneous medium, taking into account both nonlinear processes, i.e., decay and scattering. The present paper is, therefore, complementary to existing 
works in the literature. However, the novelty and importance of the present investigation is that we solve the complete weakturbulence equation including both nonlinear wave-wave (decay) as well as nonlinear wave-particle (scattering) processes, in the context of inhomogeneous plasma, using a formulation which is quite rigorous regarding derivation from first principles, including the spontaneous emission process, and incorporating self-consistency features in form more complete than in previous analyses. As we shall discuss, we find that both nonlinear processes have comparable contributions so that it is not justifiable to ignore one process over the other.

The structure of the paper is as follows: In Section 2 we briefly describe the theoretical formulation. In Section 3 we present and discuss the results of the numerical analysis. Finally, in Section 4 we conclude the paper and comment on the results obtained.

\section{THEORETICAL FORMULATION}

The equations of weak-turbulence theory that form the basis of the present numerical study are available in the literature, and recent reformulation of the same problem has been published before (see, e.g., Yoon 2006; Ziebell et al. 2008a, 2008b). For the sake of completeness, we reproduce here the basic equations using one-dimensional approximation in velocity space. We consider an electron beam propagating along the $z$-axis, where the density gradient is supposed to exist too. We allow the waves to propagate along the $z$-axis as well, so that we may write the wave vector by $\mathbf{k}=k \mathbf{e}_{\mathbf{z}}$.

The wave kinetic equations for Langmuir $(L)$ and ion-acoustic $(S)$ waves are given in terms of the spectral wave energy density,

$$
I_{k}^{\sigma L}=\left\langle E_{L}^{\sigma 2}(k)\right\rangle, \quad \text { and } \quad I_{k}^{\sigma S}=\left\langle E_{S}^{\sigma 2}(k)\right\rangle,
$$

where $E_{L}^{\sigma}(k)$ and $E_{S}^{\sigma}(k)$ represent the spectral-wave electric field component associated with $L$ and $S$ waves, respectively, and where $\sigma= \pm 1$ represents the sign of the wave phase velocity, $\sigma=+1$ denoting the direction parallel to the beam and $\sigma=-1$ denoting the anti-parallel direction.

We define a dimensionless coordinate $s$, that stands for the distance normalized to the solar radius,

$$
s=\frac{z}{R_{\odot}} .
$$

Let us define the electron plasma frequency and electron thermal velocity at a given position $s_{*}$ by

$$
\omega_{*}=\omega_{\mathrm{pe}}\left(s_{*}\right) \quad \text { and } \quad v_{*}=v_{e}\left(s_{*}\right)
$$

and use these variables to define non-dimensional quantities,

$$
\tau=\omega_{*} t, \quad u=\frac{v}{v_{*}}, \quad z=\frac{\omega}{\omega_{*}}, \quad \text { and } \quad q=\frac{k v_{*}}{\omega_{*}} .
$$

The above quantities represent dimensionless time, velocity, wave frequency, and dimensionless wave number, respectively. Here, $v$ is the velocity component along the $z$-axis, $\mathbf{v}=v \mathbf{e}_{\mathbf{z}}$.

We also define the normalized wave intensity of mode $\alpha$ and electron distribution normalized with respect to $u$ as follows:

$$
W_{\alpha}^{\sigma}(q)=(2 \pi)^{2} g_{*} \frac{I_{k}^{\sigma \alpha}}{m_{e} v_{*}^{2}}, \quad \Phi_{e}(u)=v_{*} F_{e}(v),
$$

where $g_{*}$ is proportional to the plasma parameter,

$$
g_{*}=\frac{1}{2^{3 / 2}(4 \pi)^{2} n_{*} \lambda_{*}^{3}},
$$

and $\lambda_{*}$ is the Debye length at position $s_{*}, \lambda_{*}=v_{*} /\left(\sqrt{2} \omega_{*}\right)$.

In terms of these non-dimensional variables, the wave kinetic equations for $L$ and $S$ modes are given by

$$
\begin{aligned}
& \frac{\partial W_{L}^{\sigma}(q)}{\partial \tau}+\sigma u_{L}^{g}(q) \frac{\sqrt{2} \lambda_{*}}{R_{\odot}} \frac{\partial W_{L}^{\sigma}(q)}{\partial s}-\sigma \frac{\sqrt{2} \lambda_{*}}{R_{\odot}} \frac{\partial W_{L}^{\sigma}(q)}{\partial q} \frac{\partial z_{L}(q)}{\partial s} \\
& =\frac{\pi}{q^{2}} \frac{n_{e}}{n_{*}} \int d u \delta\left[\sigma z_{L}(q)-q u\right]\left(\frac{n_{e}}{n_{*}} g_{*} \Phi_{e}(u)\right. \\
& \left.+\sigma z_{L}(q) W_{L}^{\sigma}(q) q \frac{\partial \Phi_{e}(u)}{\partial u}\right)+2 \frac{n_{*}}{n_{e}}\left(\frac{n_{*}}{n_{e}} \frac{T_{*}}{T_{e}}\right)^{1 / 2} \\
& \times \sum_{\sigma^{\prime}, \sigma^{\prime \prime}= \pm 1} \sigma z_{L}(q) \int d q^{\prime} \frac{\mu\left(q-q^{\prime}\right)}{\left|q-q^{\prime}\right|^{2}} \delta\left[\sigma z_{L}(q)\right. \\
& \left.-\sigma^{\prime} z_{L}\left(q^{\prime}\right)-\sigma^{\prime \prime} z_{S}\left(q-q^{\prime}\right)\right]\left[\sigma z_{L}(q) W_{L}^{\sigma^{\prime}}\left(q^{\prime}\right) W_{S}^{\sigma^{\prime \prime}}\right. \\
& \times\left(q-q^{\prime}\right)-\sigma^{\prime} z_{L}\left(q^{\prime}\right) W_{L}^{\sigma^{\prime \prime}}\left(q-q^{\prime}\right) W_{L}^{\sigma}(q)-\sigma^{\prime \prime} z_{L} \\
& \left.\times\left(q-q^{\prime}\right) W_{L}^{\sigma^{\prime}}\left(q^{\prime}\right) W_{L}^{\sigma}(q)\right]-\frac{n_{*}}{n_{e}} \sum_{\sigma^{\prime}= \pm 1} \int d q^{\prime} \int d u \delta \\
& \times\left[\sigma z_{L}(q)-\sigma^{\prime} z_{L}\left(q^{\prime}\right)-\left(q-q^{\prime}\right) u\right]\left[g_{*} \sigma z_{L}(q)\right. \\
& \times\left[\sigma^{\prime} z_{L}\left(q^{\prime}\right) W_{L}^{\sigma}(q)-\sigma z_{L}(q) W_{L}^{\sigma^{\prime}}\left(q^{\prime}\right)\right]\left[\boldsymbol{\Phi}_{e}(u)+\boldsymbol{\Phi}_{i}(u)\right] \\
& +W_{L}^{\sigma^{\prime}}\left(q^{\prime}\right) W_{L}^{\sigma}(q)\left(q-q^{\prime}\right) \frac{\partial}{\partial u}\left(\left[\sigma z_{L}(q)-\sigma^{\prime} z_{L}\left(q^{\prime}\right)\right]\right. \\
& \left.\left.\times \Phi_{e}(u)-\frac{m_{e}}{m_{i}} \sigma z_{L}(q) \boldsymbol{\Phi}_{i}(u)\right)\right], \\
& \frac{\partial W_{S}^{\sigma}(q)}{\partial \tau}+\sigma u_{S}^{g}(q) \frac{\sqrt{2} \lambda_{*}}{R_{\odot}} \frac{\partial W_{S}^{\sigma}(q)}{\partial s}-\sigma \frac{\sqrt{2} \lambda_{*}}{R_{\odot}} \frac{\partial W_{S}^{\sigma}(q)}{\partial q} \frac{\partial z_{S}(q)}{\partial s} \\
& =\frac{\pi \mu_{q}}{q^{2}}\left(\frac{T_{e}}{T_{*}}\right)^{2}\left(\frac{n_{*}}{n_{e}} \frac{T_{*}}{T_{e}}\right)^{1 / 2} \int d u \delta\left[\sigma z_{S}(q)-q u\right] \\
& \times\left[\frac{n_{e}}{n_{*}} g_{*}\left[\boldsymbol{\Phi}_{e}(u)+\boldsymbol{\Phi}_{i}(u)\right]+\sigma z_{L}(q) W_{S}^{\sigma}(q) q \frac{\partial}{\partial u}\right. \\
& \left.\times\left(\Phi_{e}(u)+\frac{m_{e}}{m_{i}} \boldsymbol{\Phi}_{i}(u)\right)\right]+\frac{n_{*}}{n_{e}}\left(\frac{n_{*}}{n_{e}} \frac{T_{*}}{T_{e}}\right)^{1 / 2} \\
& \times \sum_{\sigma^{\prime}, \sigma^{\prime \prime}= \pm 1} \sigma z_{L}(q) \frac{\mu(q)}{q^{2}} \int d q^{\prime} \delta\left[\sigma z_{S}(q)-\sigma^{\prime} z_{L}\left(q^{\prime}\right)\right. \\
& \left.-\sigma^{\prime \prime} z_{L}\left(q-q^{\prime}\right)\right]\left[\sigma z_{L}(q) W_{L}^{\sigma^{\prime}}\left(q^{\prime}\right) W_{L}^{\sigma^{\prime \prime}}\left(q-q^{\prime}\right)\right. \\
& -\sigma^{\prime} z_{L}\left(q^{\prime}\right) W_{L}^{\sigma^{\prime \prime}}\left(q-q^{\prime}\right) W_{S}^{\sigma}(q)-\sigma^{\prime \prime} z_{L}\left(q-q^{\prime}\right) \\
& \left.\times W_{L}^{\sigma^{\prime}}\left(q^{\prime}\right) W_{S}^{\sigma}(q)\right]
\end{aligned}
$$

where

$$
\begin{aligned}
\mu(q) & =\frac{q^{3}}{2^{3 / 2}} \sqrt{\frac{m_{e}}{m_{i}}}\left(1+\frac{3 T_{i}}{T_{e}}\right)^{1 / 2}, \\
u_{L, S}^{g}(q) & =\frac{v_{L, S}^{g}(q)}{v_{*}} \\
v_{L, S}^{g}(q) & =\frac{\partial \omega_{L, S}(k)}{\partial k} .
\end{aligned}
$$


In the above, $u_{L, S}^{g}(q)$ represents the magnitude of the normalized group velocities for $L$ and $S$ waves, $v_{L, S}^{g}(q)$ being the group speed in original definition.

The dispersion relations for $L$ and $S$ modes also appear in Equations (1) and (2). They are given by well-known expressions, which in normalized form appear as follows:

$$
\begin{aligned}
& z_{L}(q)=\frac{\omega_{p e}}{\omega_{*}}\left(1+\frac{3}{2} \frac{v_{e}^{2}}{v_{*}^{2}} \frac{\omega_{*}^{2}}{\omega_{p e}^{2}} q^{2}\right)^{1 / 2}, \\
& z_{S}(q)=\frac{q A\left(T_{e} / T_{*}\right)^{1 / 2}}{\left[1+\left(q^{2} / 2\right)\left(T_{e} / T_{*}\right)\left(n_{*} / n_{e}\right)\right]^{1 / 2}},
\end{aligned}
$$

where

$$
A=\frac{1}{\sqrt{2}}\left(\frac{m_{e}}{m_{i}}\right)^{1 / 2}\left(1+3 \frac{T_{i}}{T_{e}}\right)^{1 / 2}
$$

The normalized group velocities for $L$ and $S$ waves are given by

$$
\begin{aligned}
& u_{L}^{g}(q)=\frac{3 q}{2}\left(1+\frac{3}{2} \frac{T_{e}}{T_{*}} \frac{n_{*}}{n_{e}} q^{2}\right)^{-1 / 2}\left(\frac{n_{*}}{n_{e}}\right)^{1 / 2}\left(\frac{T_{e}}{T_{*}}\right), \\
& u_{S}^{g}(q)=A\left(1+\frac{1}{2} \frac{T_{e}}{T_{*}} \frac{n_{*}}{n_{e}} q^{2}\right)^{-3 / 2}\left(\frac{T_{e}}{T_{*}}\right)^{1 / 2} .
\end{aligned}
$$

We also need the space derivative of the dispersion relation. For $L$ and $S$ waves we have, respectively,

$$
\begin{aligned}
\frac{\partial z_{L}(q)}{\partial s} & \simeq \frac{1}{2}\left(\frac{n_{e}}{n_{*}}\right)^{-1 / 2} \frac{\partial}{\partial s}\left(\frac{n_{e}}{n_{*}}\right), \\
\frac{\partial z_{S}(q)}{\partial s} & \simeq \frac{q^{3}}{4} A\left(\frac{T_{e}}{T_{*}}\right)^{3 / 2}\left(\frac{n_{e}}{n_{*}}\right)^{-2} \frac{\partial}{\partial s}\left(\frac{n_{e}}{n_{*}}\right) .
\end{aligned}
$$

The first terms on the right-hand sides of Equations (1) and (2) describe the spontaneous and induced emissions. The induced emission term is equivalent to quasi-linear effects. The second terms contain the wave energy and momentum conservation condition, $\delta\left(\sigma \omega_{k}^{L}-\sigma^{\prime} \omega_{k^{\prime}}^{L}-\sigma^{\prime \prime} \omega_{k-k^{\prime}}^{S}\right)$ for $L$ mode and a similar three-wave resonance condition for $S$ mode. These terms describe the three-wave decay process. The papers by $\mathrm{Li}$ et al. $(2003,2006 a, 2006 b)$ retain only these terms in the wave kinetic equations.

The third term on the right-hand side of Equation (1) contains the nonlinear wave-particle resonance condition $\delta\left[\sigma \omega_{k}^{L}-\right.$ $\left.\sigma^{\prime} \omega_{k^{\prime}}^{L}-\left(k-k^{\prime}\right) v\right]$. The term dictated by this resonance condition describes the spontaneous and induced scattering of $L$ waves mediated by thermal ions. In the early work by Muschietti \& Dum (1991) only this term is retained in the nonlinear term.

The complete numerical analysis, containing both decay and scattering term, was first performed by Ziebell et al. (2001) for one-dimensional uniform plasmas and by Kontar \& Pécseli (2002) incorporating inhomogeneity effects. Extension to two dimensions in uniform plasmas was done by Ziebell et al. (2008a, 2008b) and Pavan et al. (2009). Equations (1) and (2) extend the work by Ziebell et al. (2001) in a different way in that we now include inhomogeneous effects. As already noted, the present work complements the papers by Kontar \& Pécseli (2002) and by Li et al. (2003, 2006a, 2006b), who also undertook the analysis of beam-plasma instability taking into account spatial inhomogeneity, including only the decay terms as nonlinear effects, as in Li et al. (2003, 2006a, 2006b), and decay and scattering terms, as in Kontar \& Pécseli (2002).

The term corresponding to scattering effects in Equation (2) has been neglected, since the scattering processes involving $S$ waves are extremely slow processes. In our earlier paper (Ziebell et al. 2001) as well as in a series of unpublished tests, we have exhaustively verified that for a wide range of input physical parameters of practical interests, the intensity of ion-sound turbulence is much lower than that of Langmuir turbulence. Since the ion-sound scattering process is proportional to the square of the ion-sound turbulence, it is therefore very inefficient and not expected to be effective in the timescale of our analysis. The same argument has also been used in Equation (2) to ignore the contribution of three-wave decay processes involving three ion-sound waves. Although decay processes involving three acoustic waves can be important in some contexts, such as in the analysis of acoustic turbulence (L'vov et al. 1997), they become irrelevant in comparison with other types of terms included in Equation (2), owing to the low intensity of the ionsound turbulence. On the left-hand side of Equations (1) and (2), the second term is the so-called convective term, while the third term is associated with the effects of spatial dispersion of the waves.

The evolution of the electron distribution function is given by the following Fokker-Planck equation:

$$
\begin{aligned}
\frac{\partial \Phi_{e}(u)}{\partial \tau} & +u_{z} \frac{\sqrt{2} \lambda_{*}}{R_{\odot}} \frac{\partial \Phi_{e}(u)}{\partial s} \\
& =\frac{\partial}{\partial u}\left(A_{e}(u) \Phi_{e}(u)+D_{e}(u) \frac{\partial \Phi_{e}(u)}{\partial u}\right), \\
A_{e}(u) & =g_{*} \int d q \frac{1}{q} \sum_{\sigma= \pm 1} \sigma z_{L}(q) \delta\left[\sigma z_{L}(q)-q u\right], \\
D_{e}(u) & =\int d q \sum_{\sigma= \pm 1} \delta\left[\sigma z_{L}(q)-q u\right] W_{L}^{\sigma}(q),
\end{aligned}
$$

where the term associated with coefficient $A_{e}$ describes the effects of spontaneous fluctuations, and the term with coefficient $D_{e}$ governs the velocity-space diffusion process. The ions are treated as quasi-stationary.

For a detailed derivation of the above equations for uniform plasmas, the reader is referred to Yoon (2006). These equations can be regarded as an updated version of the standard equations of the weak-turbulence theory, whose foundations can be traced back to the 1960s and 1970s. For more references, see the standard monographs (Kadomtsev 1965; Vedenov 1968; Sagdeev \& Galeev 1969; Tsytovich 1970; Davidson 1972; Thornhill \& Ter Haar 1978; Melrose 1980; Sitenko 1982). The papers Yoon (2000, 2005 and references therein) and Cairns (1987).

The initial electron distribution function at each position $s$ is assumed to be made of Maxwellian background and tenuous beam distributions. In earlier similar studies by Li et al. (2003, 2006a, 2006b), the authors investigated the time-of-flight effects of initially spatially confined electron beam moving through either uniform background plasma or through density gradient. In the present discussion, we are interested in a different initial configuration. Instead of investigating the dynamical evolution of initially spatially confined beam, we are interested in a situation where a length of flux tube (or one-dimensional density structure) is filled with electron stream. This configuration might be relevant to a quasi-steady state situation instead of a highly 
bursty situation. In one dimension, the initial velocity space distribution is given as follows:

$$
\begin{array}{r}
\Phi_{e}(s, 0)=\left(1-\frac{n_{b}}{n_{e}}\right) \frac{1}{\sqrt{\pi}}\left(\frac{T_{*}}{T_{e}}\right)^{1 / 2} \exp \left(-\frac{T_{*}}{T_{e}}\left(u-u_{0}\right)^{2}\right) \\
+\frac{n_{b}}{n_{e}} \frac{1}{\sqrt{\pi}}\left(\frac{T_{*}}{T_{b}}\right)^{1 / 2} \exp \left(-\frac{T_{*}}{T_{b}}\left(u-u_{b}\right)^{2}\right) .
\end{array}
$$

Here, $T_{e}$ and $T_{b}$ are temperatures for the background and the beam components, respectively, $u_{0}$ and $u_{b}$ are the drift speeds associated with each component, and $n_{e}$ and $n_{b}$ define the number densities for each species. The background drift velocity $u_{0}$ is introduced in our model, although it is small, so that a zero net drift velocity for the entire electron distribution as a whole is guaranteed. That is, we allow the background species to have a slight backward drift given by

$$
u_{0}=-\frac{n_{b} u_{b}}{n_{e}}
$$

Time-stationary ion distribution in one dimension is simply given by

$$
\Phi_{i}(s)=\frac{1}{\sqrt{\pi}}\left(\frac{m_{i}}{m_{e}} \frac{T_{*}}{T_{i}}\right)^{1 / 2} \exp \left(-\frac{m_{i}}{m_{e}} \frac{T_{*}}{T_{i}} u^{2}\right),
$$

where $T_{i}$ and $m_{i}$ are the ion temperature and the proton mass, respectively. In our model, the spatial dependence is introduced through inhomogeneous densities $n_{e}(s)$ and $n_{b}(s)$. We assume exponential dependence of the density ratio $n_{e} / n_{*}$,

$$
\frac{n_{e}}{n_{*}}=\exp \left(-\frac{s-s_{*}}{L_{s}}\right)
$$

where $L_{s}$ is the scale length of the spatial inhomogeneity. The temperatures are assumed to be uniform along the density structure.

The wave intensities at each position $s$ are initialized by balancing the spontaneous and induced emissions, taking into account the background population:

$$
\begin{aligned}
& W_{L}^{\sigma}(q, s, 0)=g_{*} \frac{n_{e}}{n_{*}} \frac{T_{e}}{T_{*}} \frac{1}{2\left[z_{L}(q)\right]^{2}} \\
& W_{S}^{\sigma}(q, s, 0)=\frac{g_{*}}{2 z_{L}(q) z_{S}(q)} \frac{n_{e}}{n_{*}} \frac{T_{e}}{T_{*}} \\
& \times \frac{\exp \left(-\frac{T_{*}}{T_{e}} \frac{\left(z_{q}^{S}\right)^{2}}{q^{2}}\right)+\left(\frac{m_{i}}{m_{e}} \frac{T_{e}}{T_{i}}\right)^{1 / 2} \exp \left(-\frac{m_{i}}{m_{e}} \frac{T_{*}}{T_{i}} \frac{\left(z_{q}^{S}\right)^{2}}{q^{2}}\right)}{\exp \left(-\frac{T_{*}}{T_{e}} \frac{\left(z_{q}^{S}\right)^{2}}{q^{2}}\right)+\frac{T_{e}}{T_{i}}\left(\frac{m_{i}}{m_{e}} \frac{T_{e}}{T_{i}}\right)^{1 / 2} \exp \left(-\frac{m_{i}}{m_{e}} \frac{T_{*}}{T_{i}} \frac{\left(z_{q}^{S}\right)^{2}}{q^{2}}\right)}
\end{aligned}
$$

\section{NUMERICAL ANALYSIS}

The set of Equations (1)-(5) has been solved with the use of the "splitting method" both for the particle and for the wave equation. For the particle equation, we used as boundary conditions in the velocity space the initial value of the derivative. At the extremes of the space coordinate, we used null derivative for the distribution function. For the wave equations, we used linear projection of the spectrum at the extremes of $q$, and wrote equations for all points of the space intervals, using forward and backward approximations for the space derivatives at the extremes of the space coordinate.

We employed a grid with 61 points for $q$ in the range $0<q<0.6$. For the velocity, we used 101 points for $u$, covering the velocity range $-12<u<12$. We assume the beam-tothermal number density $n_{b} / n_{e}=1.0 \times 10^{-3}$ throughout the entire length of $s$, normalized beam speed $u_{b}=5.0$, equal beam-to-background temperature $T_{b} / T_{e}=1.0$, electron-toion temperature ratio $T_{e} / T_{i}=7.0$, and the plasma parameter $\left(n_{0} \lambda_{D}^{3}\right)^{-1} \simeq 5.0 \times 10^{-3}$. For the present set of input parameters, the solar radius is approximately $1.0 \times 10^{8}$ times the Debye length. We assume the space inhomogeneity, whose scale length is given by $L_{s}=30$.

To begin with, let us first ignore nonlinear terms in the wave kinetic equations and retain only quasi-linear effects (the spontaneous and induced emission terms). Figure 1 displays the normalized $L$-wave intensity in vertical logarithm scale, as a function of $q$ and $s$. Of course, we have simultaneously solved for the ion-sound wave intensity and the electron distribution, but Figure 1 only shows the $L$-mode intensity. When nonlinear effects are ignored, the $S$-mode wave intensity does not evolve in time. We have plotted the solution at several normalized time intervals: $\tau=200,500,1000$, and 2000. Note that the peak $L$-mode intensity is located near $s=1$, centered at wave number $q \simeq 0.25$, which corresponds to the resonant wave number when the beam speed is $u_{b}=5.0$. As time progresses, the wave intensity keeps increasing and also spreading out in $q$ space until around $\tau=1000$. Beyond this point, the $L$ waves near $s=1$ evolves very slowly as the system reached quasisaturation stage. It is interesting to note that the maximum wave intensity shifts to longer and longer wavelengths as $s$ increases, and that the wave intensity monotonically decreases for increasing $s$. It is also important to note that the waves propagating in the negative direction $(q<0)$ are not generated at all in the present quasi-linear approximation. However, once we reinstate the full nonlinear interaction terms, the primary $L$ mode with $q>0$ will be seen to get back scattered by combined decay and nonlinear scattering processes so that $q<0$ modes will be generated.

In Figure 2 we show the normalized $L$-wave intensity in the same format as Figure 1, except that now we reinstate the nonlinear terms in the wave kinetic equation. As one can see, Figure 2 shows that the forward (i.e., $q>0$ ) component of the $L$-mode intensity appears largely similar to the quasi-linear approximation, but we now see that backward-propagating $L$-mode waves are generated as a result of combined decay and scattering processes. The generation of backward $L$ modes is important since the merging of forward and backward $L$ modes leads to the (harmonic) plasma emission, although the emission of transverse electromagnetic radiation is not the focus of the present paper. From Figure 2, it is clear that the nonlinear processes are central to properly account for the beam-plasma interaction physics. It is interesting to note that the backward $L$-mode generation process is effective for small $s$, while for increasing $s$, for which the forward $L$ mode decreases in intensity and shifts to longer wavelengths, the backward $L$ modes are suppressed.

One of the aims of the present paper is to compare the relative importance of the decay versus scattering as the dominant nonlinear process. In the early work by Muschietti \& Dum (1991), the authors ignored the decay process, while the papers from the University of Sydney group usually ignore the scattering term (Li et al. 2003, 2006a, 2006b). In the paper by Ziebell et al. (2001), in which the authors considered uniform plasmas, they demonstrated, by artificially turning one term versus the other in their numerical solution and comparing the outcome, that the decay and scattering processes make comparable 

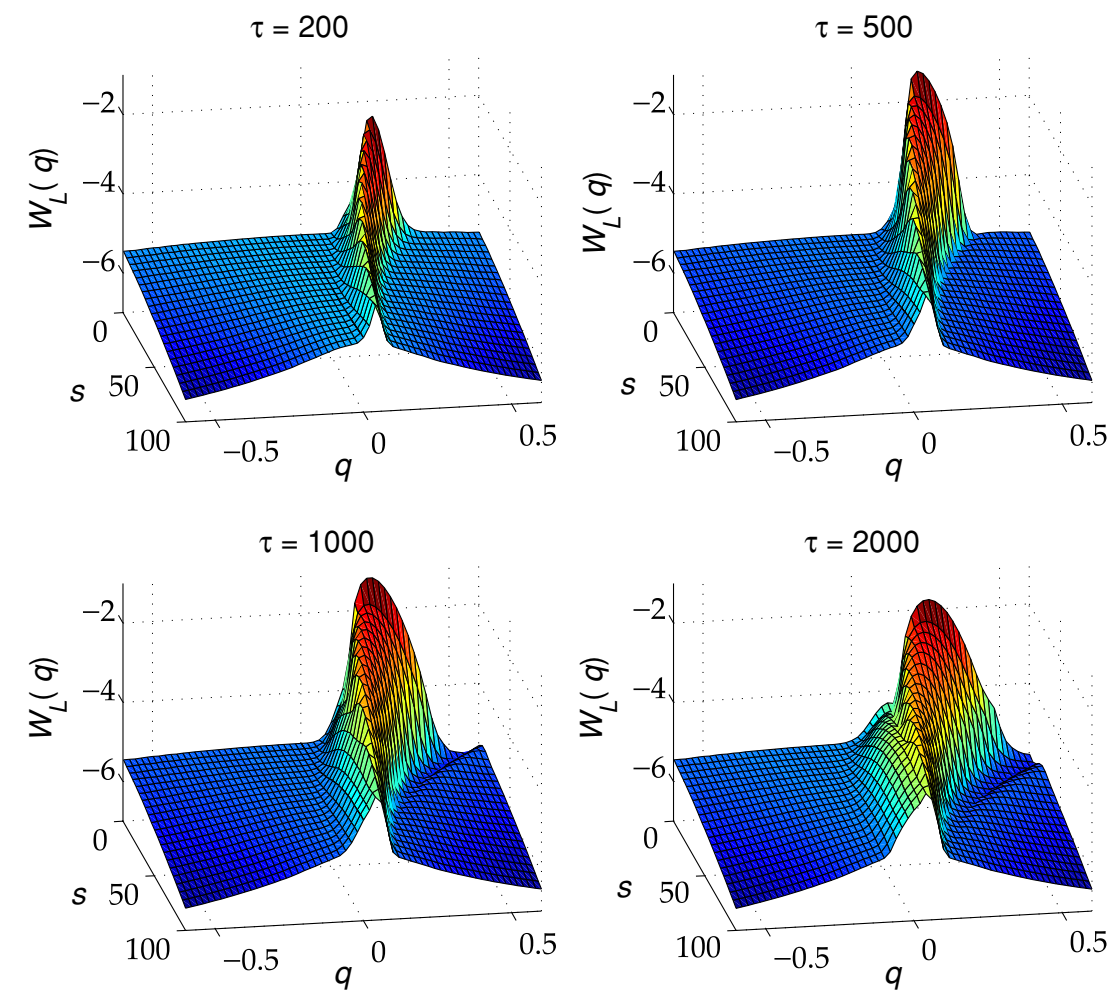

Figure 1. Normalized $L$-wave intensity, in vertical logarithm scale, as a function of $q$ and $s$, when only quasi-linear processes (spontaneous and induced emission terms) are taken into account.

(A color version of this figure is available in the online journal.)
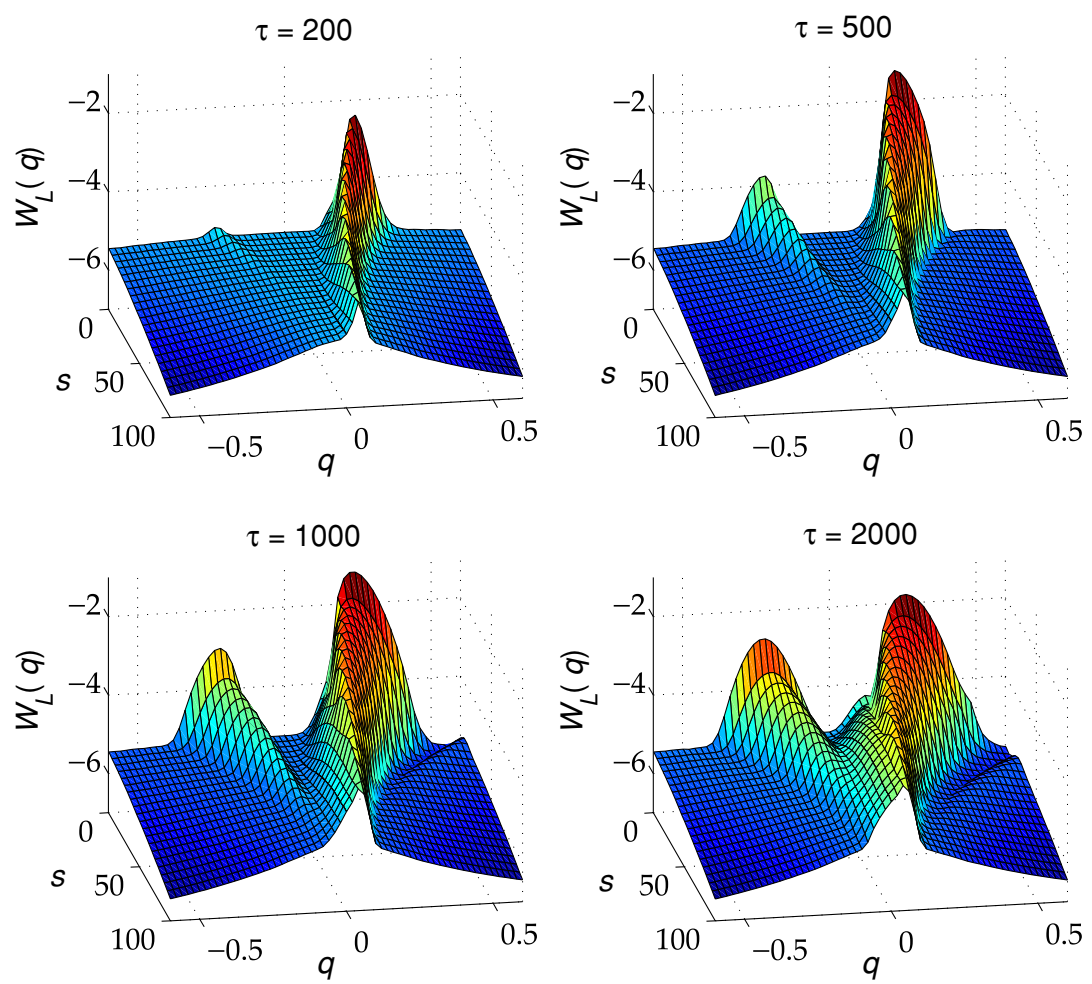

Figure 2. Normalized $L$-wave intensity, in vertical logarithm scale, as a function of $q$ and $s$, when nonlinear (decay and scattering) terms are considered in addition to quasi-linear terms.

(A color version of this figure is available in the online journal.)

contributions to the back scattering of primary Langmuir waves. Here we repeat the same analysis, except that now we are dealing with an inhomogeneous medium. In Figure 3, we thus show the normalized $L$-wave intensity in the same format as before, computed at normalized time $\tau=2000$, when only quasilinear processes are considered (top left), when only the decay 

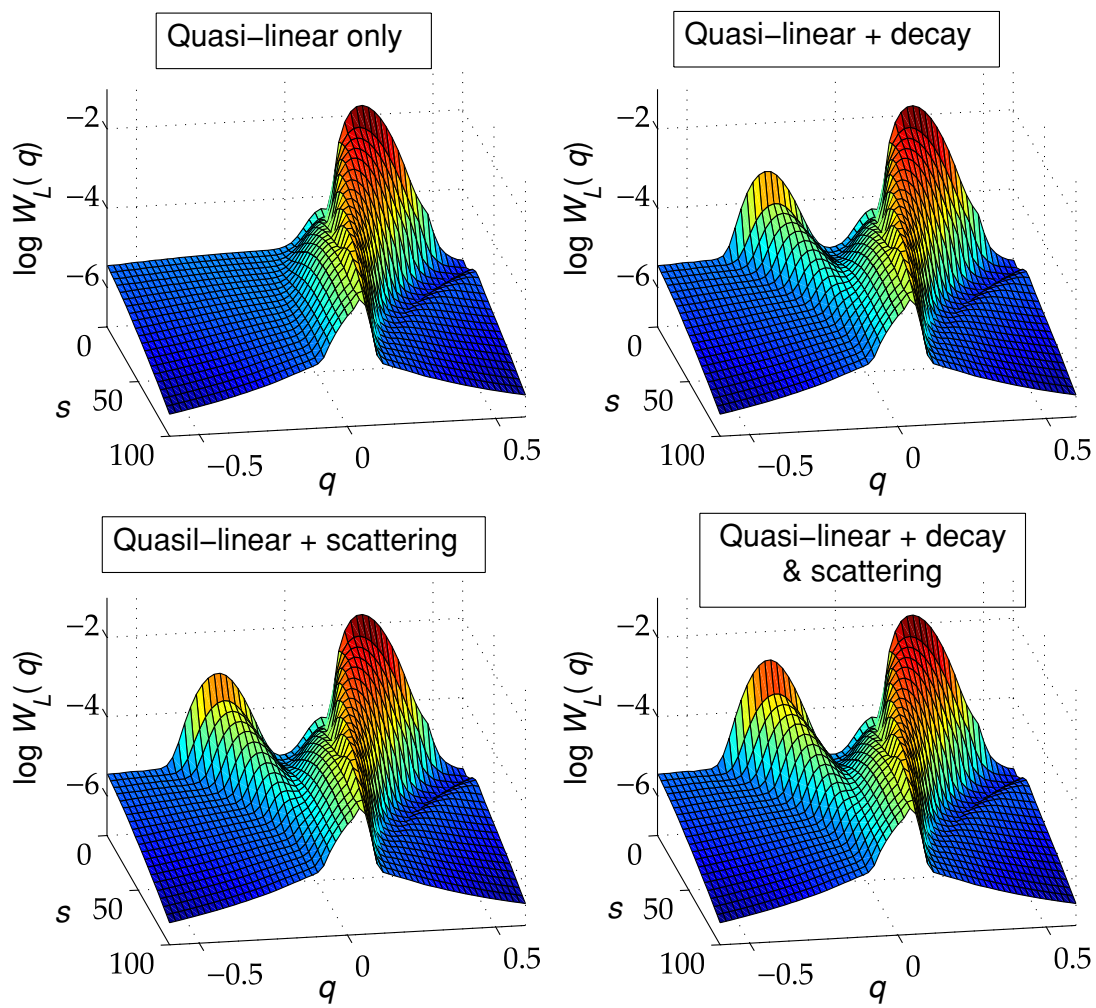

Figure 3. Comparison of various approximations: normalized $L$-mode intensity at $\tau=2000$ when only quasi-linear processes are considered (top left), when only the decay process is added (top right), when only the scattering term is added (bottom left), and when fully nonlinear (decay and scattering) terms are considered in addition to quasi-linear terms (bottom right).

(A color version of this figure is available in the online journal.)
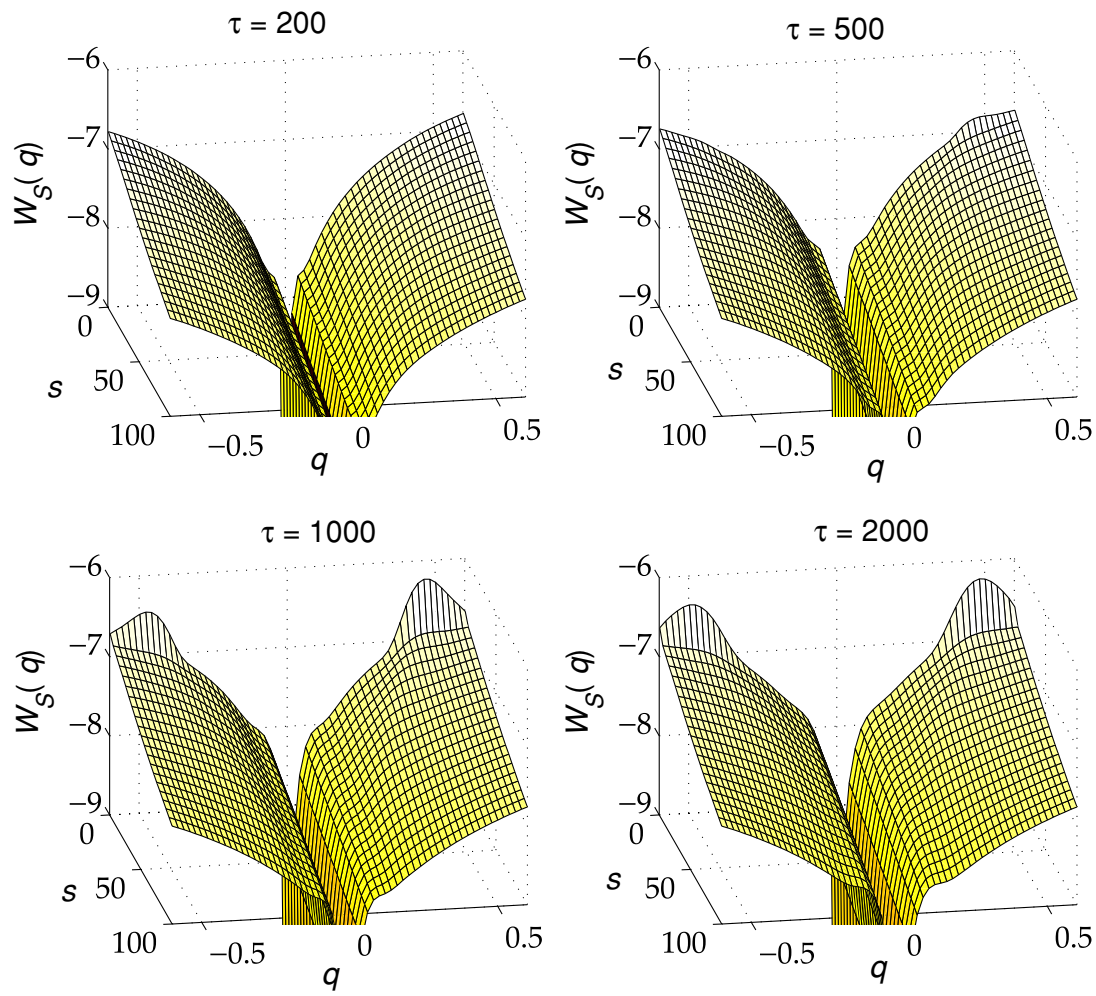

Figure 4. Normalized $S$-wave intensity, in vertical logarithm scale, as a function of $q$ and $s$. (A color version of this figure is available in the online journal.)

process is added to the quasi-linear terms (top right), when only the scattering term is added to quasi-linear terms while artifi- cially turning the decay terms off (bottom left), and when both decay and scattering terms (fully nonlinear result) are consid- 

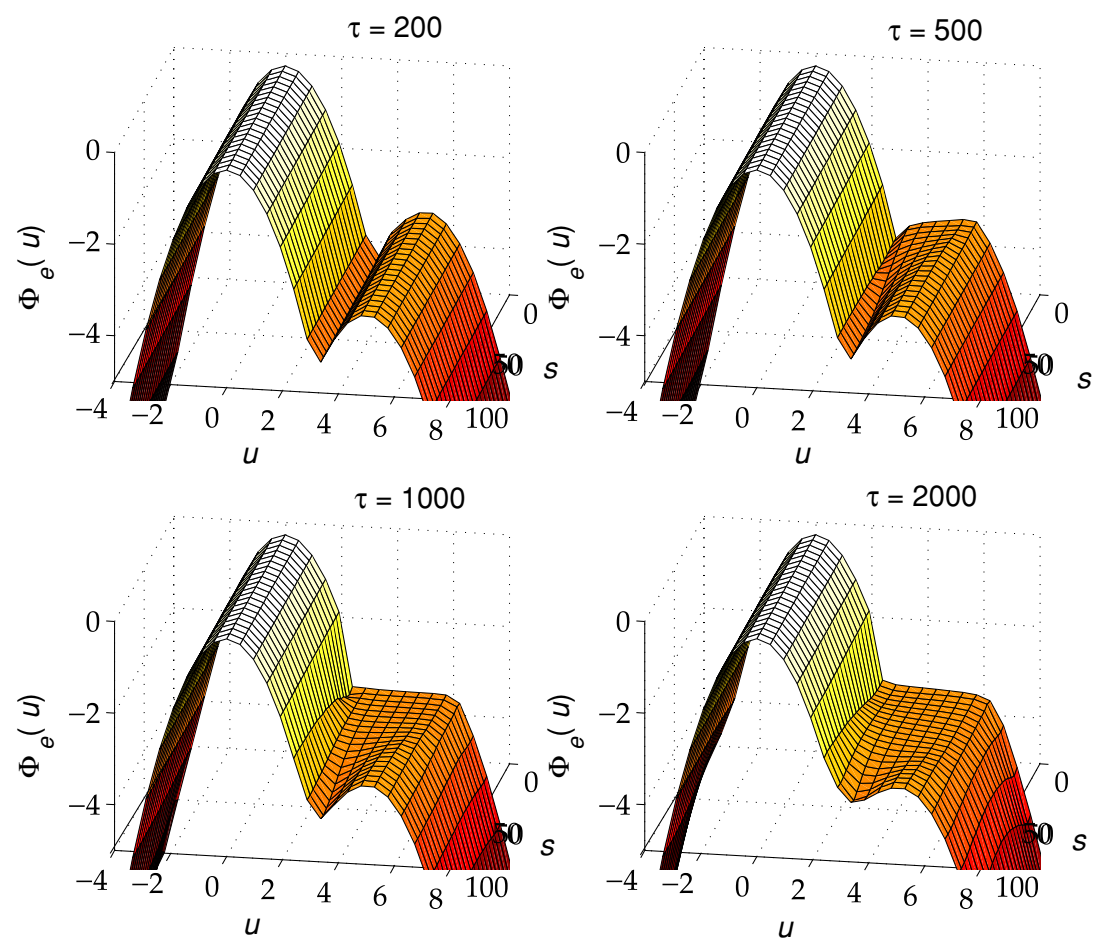

Figure 5. Normalized electron velocity distribution function, in vertical logarithm scale, as a function of $q$ and $s$.

(A color version of this figure is available in the online journal.)

ered in addition to quasi-linear terms (bottom right). As one can see, the forward-propagating (primary) Langmuir waves are adequately described by quasi-linear theory alone. However, it takes both the decay and scattering terms to completely account for the back scattered $L$ mode with $q<0$. As a matter of fact, if anything, the scattering term seems to produce slightly higher back scattered $L$-mode intensity when compared with the decay only solution. From this, we conclude that, at least for the present set of input parameters, the three-wave decay and nonlinear wave-particle (scattering) processes are equally important for the generation of backward-propagating Langmuir waves during the beam-plasma interaction process.

In obtaining the numerical solution for $L$-mode intensity, we have also concomitantly solved for the ion-sound wave intensity and the electron velocity distribution function. In Figure 4, we display the normalized $S$-wave intensity, in vertical logarithm scale, as a function of $q$ and $s$. Here, of course, we have considered the full nonlinear terms in the wave kinetic equations. The ion-sound mode intensity does not show much appreciable change above and beyond the initially imposed level of spontaneous emission. Nevertheless, this does not mean that the ion-sound mode is unimportant. Without the $S$ mode, the three-wave decay process is not possible in the first place. This means that, although the ion-acoustic waves may not be prominent, they nevertheless play an important intermediary role in the dynamical sense. Often, the level of observed ionsound wave signature is used as evidence for decay processes in the literature. However, the present result shows that caution must be exercised in that regard. The lack of observable ionsound mode signature does not necessary mean that the threewave decay processes are absent.

In Figure 5 we show the normalized electron distribution function, in vertical logarithm scale, as a function of $u$ and $s$. At $\tau=200$, it can be seen that the region of positive derivative associated with the beam component is still present. At $\tau=500$, a significant platea $u$ appears in small-s region. At $\tau=1000$ and 2000 , one can observe that the plateau formation that took place for small $s$ at early times, gradually spreads out to large-s region.

\section{CONCLUSION}

The problem of an electron beam propagating in inhomogeneous solar wind plasma is important in view of the solar type II and type III radio bursts. Many scientists in the past addressed this issue by means of quasi-linear analysis (Vedenov et al. 1967; Takakura \& Shibahashi 1976; Magelssen \& Smith 1977; Grognard 1985; Kontar 2001; Li et al. 2002; Khalilpour et al. 2009). However, in order to fully characterize the nonlinear dynamics, one must go beyond quasi-linear approximation and employ weak-turbulence theory.

The numerical solution of the weak-turbulence equation by Muschietti \& Dum (1991) included only the induced scattering process, while Li et al. $(2003,2006 \mathrm{a}, 2006 \mathrm{~b})$ relied only on the decay process. The works by Ziebell et al. (2001) as well as more general later works by Ziebell et al. (2008a, 2008b) and by Pavan et al. (2009) do include both decay and scattering processes but ignored the spatial inhomogeneity. Kontar \& Pécseli introduced inhomogeneity effects including both decay and scattering processes, but the description of quasi-linear and nonlinear effects was more approximate and less self-consistent than in Ziebell et al. (2001, 2008a, 2008b) and in Pavan et al. (2009).

In the present paper, we have solved a self-consistent system of weak-turbulence equations, including both decay and scattering processes, in an inhomogeneous medium. On the basis of detailed comparisons of the influence of each term in the nonlinear wave kinetic equation (see Figure 3), we concluded that quasi-linear effect is sufficient to characterize the generation of forward-propagating primary Langmuir waves. However, as far as the backward-propagating Langmuir wave is concerned, 
which is important for eventual radiation generation, we found that nonlinear decay and scattering processes make comparable contributions. This finding may be important in the quantitative analysis of plasma emission process with application to solar type II and/or type III radio bursts.

In the future, we intend to make specific application of the present finding in investigating the actual radio emission process. In order to carry out such a purpose, one must first generalize the present analysis to two dimensions, and include electromagnetic effects. Such a task is, of course, very demanding in terms of both theoretical and numerical efforts, but it shall be the subject of our future research.

This work has been partially supported by the Brazilian agencies CNPq, FAPERGS, and CAPES. J.P. acknowledges the hospitality of the University of Maryland. P.H.Y. acknowledges NSF grant AGS0940985 to the University of Maryland, AFOSR contract FA9550-07-0053 to MTL, and WCU grant R31-10016 from the Korean Ministry of Education, Science and Technology to Kyung Hee University, Korea.

\section{REFERENCES}

Appert, K., Tran, T. M., \& Vaclavik, J. 1976, Phys. Rev. Lett., 37, 502

Cairns, I. H. 1987, J. Plasma Phys., 38, 169

Davidson, R. C. 1972, Methods in Nonlinear Plasma Theory (New York: Academic)

Goldman, M. V. 1983, Sol. Phys., 89, 403

Grognard, R. J. M. 1985, in Solar Radiophysics, ed. D. J. McLean \& N. R. Labrum (Cambridge: Cambridge Univ. Press), 253

Ishihara, O., \& Hirose, A. 1981, Phys. Rev. Lett., 46, 771

Ishihara, O., \& Hirose, A. 1983a, Phys. Fluids, 26, 100

Ishihara, O., \& Hirose, A. 1983b, Phys. Rev. Lett., 50, 1783
Kadomtsev, B. B. 1965, Plasma Turbulence (New York: Academic)

Khalilpour, H., Foroutan, G., Moslehi-Fard, M., Li, B., \& Robinson, P. A. 2009, Phys. Plasmas, 16, 072901

Kontar, E. P. 2001, Sol. Phys., 202, 131

Kontar, E. P., \& Pécseli, H. L. 2002, Phys. Rev. E, 65, 066408

Li, B., Robinson, P. A., \& Cairns, I. H. 2002, Phys. Plasmas, 9, 2976

Li, B., Robinson, P. A., \& Cairns, I. H. 2006a, Phys. Rev. Lett., 96, 145005

Li, B., Robinson, P. A., \& Cairns, I. H. 2006b, Phys. Plasmas, 13, 092902

Li, B., Willes, A. J., Robinson, P. A., \& Cairns, I. H. 2003, Phys. Plasmas, 10, 2748

L'vov, V. S., L’vov, Yu., Newell, A. C., \& Zakharov, V. 1997, Phys. Rev. E, 56, 390

Magelssen, G. R., \& Smith, D. F. 1977, Sol. Phys., 55, 211

Melrose, D. B. 1980, Plasma Astrophysics (New York: Gordon and Breach)

Melrose, D. B. 1985, in Solar Radiophysics, ed. D. J. McLean \& N. R. Labrum (New York: Cambridge Univ. Press), 177

Muschietti, L., \& Dum, C. T. 1991, Phys. Fluids B, 3, 1968

Muschietti, L., Roth, I., \& Delory, G. 1997, J. Geophys. Res., 102, 27217

Pavan, J., Ziebell, L. F., Gaelzer, R., \& Yoon, P. H. 2009, J. Geophys. Res., 114, A01106

Robinson, P. A., \& Cairns, I. H. 1998a, Sol. Phys., 181, 363

Robinson, P. A., \& Cairns, I. H. 1998b, Sol. Phys., 181, 395

Robinson, P. A., \& Cairns, I. H. 1998c, Sol. Phys., 181, 429

Sagdeev, R. Z., \& Galeev, A. A. 1969, Nonlinear Plasma Theory (New York: Benjamin)

Sitenko, A. G. 1982, Fluctuations and Nonlinear Wave Interactions in Plasmas (New York: Pergamon)

Takakura, T., \& Shibahashi, H. 1976, Sol. Phys., 46, 323

Thornhill, S. G., \& Ter Haar, D. 1978, Phys. Rep., 43, 44

Tsytovich, V. N. 1970, Nonlinear Effects in a Plasma (New York: Plenum)

Vedenov, A. A. 1968, Theory of Turbulent Plasma (New York: Elsevier)

Vedenov, A. A., Gordeev, A. V., \& Rudakov, L. I. 1967, Plasma Phys., 9, 719

Yoon, P. H. 2000, Phys. Plasmas, 7, 4858

Yoon, P. H. 2005, Phys. Plasmas, 12, 042306

Yoon, P. H. 2006, Phys. Plasmas, 13, 022302

Ziebell, L. F., Gaelzer, R., Pavan, J., \& Yoon, P. H. 2008a, Plasma Phys. Control. Fusion, 50, 085011

Ziebell, L. F., Gaelzer, R., \& Yoon, P. H. 2001, Phys. Plasmas, 8, 3982

Ziebell, L. F., Gaelzer, R., \& Yoon, P. H. 2008b, Phys. Plasmas, 15, 032303 